\title{
Inducción de enraizamiento en estacas de Berberidopsis corallina con ácido indolbutírico
}

\author{
Induction of rooting in cuttings of Berberidopsis corallina \\ with indol butyric acid
}

\begin{abstract}
Mirtha Latsague Vidala*, Patricia Sáez Delgado ${ }^{a}$ y Enrique Hauenstein Barra ${ }^{a}$
*Autor de correspondencia: aUniversidad Católica de Temuco, Escuela de Ciencias Ambientales, Facultad de Recursos Naturales, casilla 15-D, tel.: 56-45-205409, fax: 56-45-211034, Temuco, Chile, mlatsagu@uct.cl
\end{abstract}

\begin{abstract}
SUMMARY
Berberidopsis corallina is an endemic species from Chile, presenting conservation problems and being catalogued as "endangered" species. Its persistent diminution is associated to cutting and competition with Eucalyptus spp. and Pinus radiata. The objective of the present work was to contribute in the management for the recovery of this species through the evaluation of its reproductive capacity by means of propagation by cuttings. For vegetative propagation, semi-woody cuttings were collected during March of 2007 in Villa Las Araucarias, Region of La Araucanía. The collected cuttings were treated with Indolbutyric acid (IBA) at different concentrations $\left(0,500,1,000\right.$ and $\left.1,500 \mathrm{mgL}^{-1}\right)$ as rooting stimulator. After five months of maintenance of cuttings in a bottom heating, an $87 \%$ of total rooting was obtained. The best result concerning the process of rizogenesis was shown by $1,000 \mathrm{mg} \mathrm{L}^{-1}$ IBA with a $90 \%$ of rooting. Besides, the highest average of root length was found in this same treatment with an average of 13.6 $\mathrm{cm}$. The greatest number of roots was obtained in $1,500 \mathrm{mgL}^{-1}$ treatment with a value average of 38.1 roots by cuttings. It is concludes that $B$. corallina can be vegetatively reproduced through the rizogenesis of cuttings treated with IBA, as a practice to contribute to the recovery of this species.
\end{abstract}

Key words: Berberidopsis corallina, IBA, semi ligneus cuttings.

\section{RESUMEN}

Berberidopsis corallina es una especie endémica de Chile que debido a su estado de conservación ha sido catalogada como especie "en peligro de extinción". Su persistente disminución ha estado asociada a tala y competencia con Eucalyptus spp. y Pinus radiata. El objetivo del presente trabajo fue contribuir en la gestión de recuperación de esta especie a través de la evaluación de su capacidad reproductiva mediante propagación por estacas. Para la propagación vegetativa se colectaron estacas semileñosas durante marzo de 2007 en el sector Villa Las Araucarias, Región de La Araucanía. Las estacas cosechadas se trataron con ácido indolbutírico (AIB) en distintas concentraciones $\left(0,500,1.000\right.$ y $\left.1.500 \mathrm{mg} \mathrm{L}^{-1}\right)$ como factor de enraizamiento. Después de cinco meses de mantenidas las estacas en cama caliente se obtuvo un $87 \%$ de enraizamiento total. La concentración de AIB de $1.000 \mathrm{mg} \mathrm{L}^{-1}$ mostró los mejores resultados respecto al proceso de rizogénesis con un $90 \%$ de enraizamiento. Además se encontró el mayor promedio de longitud de raíces en este mismo tratamiento con un promedio de $13,64 \mathrm{~cm}$. El mayor número de raíces se obtuvo en el tratamiento $1.500 \mathrm{mg} \mathrm{L}^{-1}$ con un valor promedio de 38,11 raíces por estaca. Se concluye que $B$. corallina puede ser reproducido vegetativamente a través de la rizogénesis de estacas tratadas con AIB, como una práctica para contribuir a la recuperación de esta especie.

Palabras clave: Berberidopsis corallina, AIB, estacas semileñosas.

\section{INTRODUCCIÓN}

Existen especies que son particularmente sensibles a cambios de su hábitat y son aquellas que hoy se encuentran con serios problemas de conservación, como es el caso de Berberidopsis corallina Hooker f. (michay rojo), una planta trepadora endémica de la Cordillera de la Costa, que se distribuye en forma discontinua desde la Región del Maule hasta la Región de Los Lagos, sólo por la Cordillera de la Costa ( $34^{\circ} 42^{\prime} \mathrm{S}^{\prime} 72^{\circ} 02^{\prime} \mathrm{O}$ y $\left.40^{\circ} 27^{\prime} \mathrm{S}-73^{\circ} 42^{\prime} \mathrm{O}\right)$, presentando numerosas localidades en la parte este de la Cordillera de Nahuelbuta (Hechenleitner et al. 2005). Esta especie sólo se encuentra protegida en el Sistema Nacional de Áreas Silvestres Protegidas del Estado (SNASPE), en la Reserva Nacional Los Queules, VII Región (Muñoz 1971). Benoit (1989) señala que B. corallina se encuentra en la categoría de "en peligro de extinción", lo que se refiere a poblaciones que se han reducido a nivel crítico 
o cuyo hábitat se ha reducido tan drásticamente que por ello existe el riesgo inminente de extinción (Hoffmann 1982). El estado actual de B. corallina se debe precisamente a la sustitución del bosque nativo siempreverde por plantaciones exóticas, deforestación de su hábitat natural, utilización como planta ornamental por sus llamativas flores y excesiva extracción de sus hebras para cestería (Muñoz 1971, Hauenstein 2002).

Una forma de cambiar el estado de conservación es incrementando el recurso por medio del manejo (Santelices 2005), es decir, aplicar técnicas orientadas a la repoblación de las áreas afectadas con el fin de conservar esta especie. Para ello la reproducción vegetativa -principalmente la reproducción por estacas- ha sido utilizada ampliamente en especies leñosas. A pesar de esto, existen muy pocos reportes publicados sobre la propagación de $B$. corallina y sólo es posible obtener información técnica sobre la propagación vegetativa de esta especie. Por lo anterior, el objetivo de este estudio fue evaluar la respuesta al tratamiento de enraizamiento de estacas semileñosas de $B$. corallina, como un aporte a la conservación de ésta.

\section{MÉTODOS}

La colecta de material vegetal se realizó durante marzo de 2007, a partir de guías semileñosas obtenidas desde su zona de crecimiento natural en el sector Villa Las Araucarias, al sur de la Cordillera de Nahuelbuta ( $38^{\circ} 26^{\prime}$ a $38^{\circ} 29^{\prime} \mathrm{S}$ y $73^{\circ} 12^{\prime}$ a $73^{\circ} 14^{\prime} \mathrm{O}$ ), a $40 \mathrm{~km}$ de la comuna de Carahue y a $100 \mathrm{~km}$ de la ciudad de Temuco.

Se seleccionaron estacas semilignificadas de $15 \mathrm{~cm}$ de longitud y $2 \mathrm{~mm}$ de diámetro, dejando dos hojas por estaca y un par de yemas visibles. El material vegetal fue trasladado en bolsas plásticas al invernadero de la Universidad Católica de Temuco ubicado en la ciudad de Temuco, Región de La Araucanía, en donde se realizó el ensayo.

Debido a que el ácido indolbutírico (AIB) es la auxina más usada para estimular la formación de raíces adventicias (Mesén et al. 1997, Hartmann y Kester 1998), se estudió el efecto de distintas concentraciones, aplicando cuatro tratamientos: T1 (AIB a $500 \mathrm{mg} \mathrm{L}^{-1}$ ); T2 (AIB a 1.000 $\mathrm{mg} \mathrm{L}^{-1}$ ) y T3 (AIB a $1.500 \mathrm{mg} \mathrm{L}^{-1}$ ), más un tratamiento control (T0). Se utilizaron 84 estacas por tratamiento, considerando cada estaca como una unidad experimental.

La desinfección de las estacas se hizo por inmersión de su base durante 15 minutos en una solución $10 \mathrm{~g} \mathrm{~L}^{-1}$ de un fungicida de contacto ${ }^{1}$. Luego se sumergieron en solución de AIB por 15 minutos, de acuerdo a las concentraciones de cada tratamiento.

Una vez tratadas las estacas, se instalaron en almacigueras sobre sustrato de corteza de pino compostada

1 "Captan" de la empresa ANASAC.
(G-15) pH 5,5 más perlita de $\mathrm{pH}$ neutro en proporción 3:1. Posteriormente se mantuvieron en cama caliente a una temperatura constante de $25^{\circ} \mathrm{C}$ durante cinco meses. La temperatura ambiental se controló a través de un termómetro digital y se mantuvo a $16^{\circ} \mathrm{C}$. Para mantener la humedad se programaron cuatro riegos diarios a través de un sistema de aspersores (microjet), disminuyendo a una vez o ninguna según la humedad del invernadero.

Una vez finalizado el ensayo se evaluó el porcentaje de sobrevivencia, formación de callo y porcentaje de enraizamiento. Para analizar el potencial de crecimiento radicular se cuantificó número de raíces por estaca y longitud de éstas. La validación de los resultados se realizó a través de un análisis de varianza, con un nivel de confianza de $95 \%$. Las diferencias significativas fueron determinadas mediante la prueba de comparaciones múltiples de Tukey.

\section{RESULTADOS}

Se observó un alto porcentaje de sobrevivencia en las estacas de $B$. corallina, sin existir diferencias significativas entre los tratamientos $(P>0,05)$ (cuadro 1). Respecto a la respuesta al enraizamiento, se logró inducir el desarrollo de raíces en todos los tratamientos probados, sin encontrar diferencias significativas entre las distintas concentraciones hormonales ensayadas $(P>0,05)$. De las estacas enraizadas, el $100 \%$ formó raíces directamente a partir del tallo (cuadro1).

Berberidopsis corallina produjo callos y raíces, incluso sin necesidad de aplicar AIB (cuadro 1). A pesar de que en todos los tratamientos hubo formación de callo, esto ocurrió en un bajo porcentaje de estacas, en las que no se observó formación de raíces adventicias. Respecto al potencial de crecimiento radicular, el número de raíces fue diferente entre tratamientos $(P<0,05)$, formándose los grupos homogéneos T0-T1 y T2-T3, respectivamente. El mayor número de raíces se obtuvo en el tratamiento $1.500 \mathrm{mg} \mathrm{L}^{-1}$ con un valor promedio de 38,1 raíces por estaca. El mayor promedio de longitud de raíces se observó en el tratamiento $1.000 \mathrm{mg} \mathrm{L}^{-1}$ con un promedio de $13,6 \mathrm{~cm}(P<0,05)$ (cuadro 1$)$.

\section{DISCUSIÓN}

Los resultados indican que el material vegetal tuvo buena adaptación y resistencia a las condiciones de enraizamiento, lo cual se manifiesta en el alto porcentaje de sobrevivencia y con medidas de variabilidad similares entre los distintos tratamientos, situación que explica la falta de diferencias significativas entre ellos. Según Santelices (1993), uno de los parámetros más importantes a medir en reproducción vegetativa es la sobrevivencia de las estacas, ya que para obtener un enraizamiento satisfactorio, es esencial la sobrevivencia de un gran número del material vegetal. 
Cuadro 1. Efecto de las distintas concentraciones de ácido indolbutírico en estacas de B. corallina colectadas durante marzo de 2007 (media $\pm \mathrm{S})$.

Effect of different indol butyric acid concentrations on B. corallina cuttings collected during March of 2007 (mean \pm S).

\begin{tabular}{|c|c|c|c|c|c|}
\hline \multirow[t]{2}{*}{ Tratamiento } & \multirow{2}{*}{$\begin{array}{c}\text { Sobrevivencia } \\
(\%)\end{array}$} & \multirow{2}{*}{$\begin{array}{l}\text { Formación de callo } \\
\qquad(\%)\end{array}$} & \multirow{2}{*}{$\begin{array}{c}\text { Enraizamiento } \\
(\%)\end{array}$} & \multicolumn{2}{|c|}{ Crecimiento radicular } \\
\hline & & & & Cantidad $\left(\mathrm{N}^{\circ}\right)$ & Longitud (mm) \\
\hline $\mathrm{T} 0\left(0 \mathrm{mg} \mathrm{L}^{-1}\right)$ & $56 \pm 13,6 \mathrm{a}$ & $17 \pm 8,1 \mathrm{a}$ & $83 \pm 11,6 \mathrm{a}$ & $24,9 \pm 6 \mathrm{a}$ & $80,1 \pm 3,5 \mathrm{ab}$ \\
\hline $\mathrm{T} 1\left(500 \mathrm{mg} \mathrm{L}^{-1}\right)$ & $52 \pm 16,4$ a & $11 \pm 5,3 \mathrm{a}$ & $89 \pm 7,7 \mathrm{a}$ & $25,4 \pm 9 \mathrm{a}$ & $37,6 \pm 1,2 \mathrm{a}$ \\
\hline $\mathrm{T} 2\left(1.000 \mathrm{mg} \mathrm{L}^{-1}\right)$ & $71 \pm 6,2 \mathrm{a}$ & $10 \pm 5,4 \mathrm{a}$ & $90 \pm 4,2 \mathrm{a}$ & $34,1 \pm 14 \mathrm{~b}$ & $136,5 \pm 5,2 \mathrm{~b}$ \\
\hline Т3(1.500 mg L -1$)$ & $60 \pm 10,9 \mathrm{a}$ & $16 \pm 3,5 \mathrm{a}$ & $84 \pm 7,1 \mathrm{a}$ & $38,1 \pm 16 b$ & $93,3 \pm 4,9 a b$ \\
\hline
\end{tabular}

Valores promedios con letra distinta indican diferencias significativas entre tratamientos $P<0,05$.

Averages values with different letters indicate significant differences between treatments $P<0.05$.

La falta de asociación significativa entre la formación de raíces y la presencia de hormona AIB demuestra que $B$. corallina puede propagarse a partir de estacas sin dificultad y a bajo costo. Aunque la asociación de la aplicación de la hormona y la producción de raíces no fue significativa se produjo una mejor respuesta con las dosis bajas de AIB, entre $500 \mathrm{mg} \mathrm{L}^{-1}$ y $1.000 \mathrm{mg} \mathrm{L}^{-1}$. El mayor porcentaje de enraizamiento observado en la concentración $1.000 \mathrm{mg} \mathrm{L}^{-1}$ coincide con lo señalado por Doll et al. (2003), para Buddleja globosa Hope, arbusto nativo que crece en Chile, quienes reportaron mayores porcentajes de enraizamiento en concentraciones de $1.000 \mathrm{mg} \mathrm{L}^{-1}$ con un $83 \%$ de respuesta.

Por otra parte, no se observa una tendencia a aumentar la producción de raíces con el incremento de la concentración de AIB, similar a lo informado por Bonfil-Sanders et al. (2007) para el género Bursera.

Respecto a la formación de callos, los resultados coinciden con los obtenidos por Santelices y García (2003), quienes estudiaron la influencia de diferentes concentraciones de AIB sobre la capacidad rizogénica de Nothofagus alessandrii Espinosa, informando como resultado la formación de callo en todos los tratamientos realizados sin que se produjera arraigamiento.

La formación de callos y raíces es una respuesta común en la propagación por estacas (Mateo-Sánchez et al. 1998). El hecho de que las estacas hayan llegado a formar callo es un indicador de que las condiciones en que estuvieron fueron las adecuadas para el proceso de rizogénesis. Como es sabido, ambos procesos son independientes entre sí pero requieren de condiciones similares para desarrollarse (Hartmann y Kester 1998, Santelices 1998). Es interesante indicar que las raíces se desarrollaron en toda la porción del esqueje en contacto con la hormona, indicando que no existen barreras mecánicas en los tallos para la emisión de raíces adventicias (Hartmann y Kester 1998).

Trabajos realizados por Joublan et al. (1998) en propagación vegetativa de Hippophae rhamnoides Juss demuestran que la estimulación en la formación de raíces en presencia de hormona AIB es claramente superior respecto de tratamiento sin hormona. Por su parte, Santelices y Cabello (2006) informan que estacas de N. glauca aumentan significativamente el número de raíces al ser tratadas con AIB respecto del control.

La diferencia significativa observada respecto al número de raíces frente a los distintos tratamientos difiere de los resultados obtenidos por Santelices y García (2003), quienes informan que estacas de $N$. alessandrii no presentan diferencias significativas en el número de raíces frente a los distintos tratamientos. A medida que aumenta la concentración de la solución hormonal, aumenta el número de raíces (cuadro 1), lo que difiere de los resultados publicados por Santelices y García (2003) y Santelices y Cabello (2006), quienes reportan una disminución del número de raíces al aumentar las concentraciones del tratamiento, en $N$. alessandrii y $N$. glauca, respectivamente.

Respecto de la longitud de raíces, existen diferencias significativas $(P<0,05)$ entre los distintos tratamientos. El valor promedio más alto alcanzó a $13,6 \mathrm{~cm}$, obtenido en la concentración $1.000 \mathrm{mg} \mathrm{L}^{-1}$, a diferencia de lo obtenido por Doll et al. (2003) en B. globosa, quienes al trabajar con AIB en la misma concentración obtuvieron una media de 4,9 $\mathrm{cm}$. Sin embargo, no se observa una tendencia a aumentar la longitud de las raíces a mayor concentración hormonal. Estos resultados difieren de las respuestas obtenidas por Santelices y García (2003) en N. alessandrii, quienes reportaron que a medida que aumenta la concentración hormonal mejora la longitud de las raíces, y por Melgares et al. (2002) quienes señalan que se observa una tendencia a aumentar los valores de longitud de raíces a mayores temperaturas y mayores concentraciones de AIB en Coriaria myrtifolia Linn. Los resultados obtenidos son comparables con los descritos por García et al. (2001) en la solanácea Physolis ixocarpa Brot., en que tratamientos con concentración de 1.000 y $1.500 \mathrm{mg} \mathrm{L}^{-1}$ de AIB incrementan la longitud de las raíces. 


\section{CONCLUSIONES}

Estacas semileñosas de Berberidopsis corallina cosechadas en marzo presentan un buen potencial de enraizamiento. La concentración de AIB de $1.000 \mathrm{mg} \mathrm{L}^{-1}$ mostró los mejores resultados respecto a longitud de raíces. El mayor número de raíces se obtuvo en el tratamiento $1.500 \mathrm{mg} \mathrm{L}^{-1}$ de AIB.

\section{AGRADECIMIENTOS}

Los autores agradecen al Fondo de Protección Ambiental de la Comisión Nacional del Medio Ambiente (FPA CONAMA, Chile).

\section{REFERENCIAS}

Benoit I. 1989. Libro rojo de la flora terrestre de Chile (Primera Parte). Santiago, Chile. CONAF. 157 p.

Bonfil-Sanders C, P Mendoza, J Ulloa. 2007. Enraizamiento y formación de callos en estacas de siete especies del género Bursera Agrociencia 41: 103-109.

Doll U, H Vogel, P Jeldres, M Muñoz. 2003. Estudios de propagación vegetativa en matico (Buddleja globosa). Revista Ciencia e Investigación Agraria 30: 211-216.

García L, J Jiménez, L Peña, P Rodríguez. 2001. Propagación vegetativa en tomate de cáscara (Physalis ixocarpa Brot.) mediante enraizamiento de esquejes. Agricultura Técnica México 27(1): 27-33

Hartmann H, D Kester. 1998. Propagación de Plantas, Principios y Prácticas. México. Editorial Continental. 760 p.

Hauenstein E. 2002. Notas sobre Berberidopsis corallina Hooker (Berberidopsidaceae) ¿Especie en Peligro? Gestión Ambiental (Chile) 8: 63-69.

Hechenleitner P, M Gardner, P Thomas, C Echeverría, B Escobar, P Brownless, C Martínez. 2005. Plantas Amenazadas del Centro-Sur de Chile. Distribución, Conservación y Propagación. Valdivia, Chile. Universidad Austral de Chile y Real Jardín Botánico de Edimburgo. 188 p.
Hoffmann A. 1982. Flora silvestre de Chile. Zona austral. Una guía ilustrada para la identificación de especies de plantas leñosas del sur de Chile. Santiago, Chile. Ediciones Fundación Claudio Gay. 255 p.

Joublan J, M Berti, R Wilckens. 1998. Propagación vegetativa en falso espino (Hippophae rhamnoides Juss). Agro Sur 26: 36-41.

Mateo Sánchez J, J Vargas Hernández, M López Peralta, J Jasso Mata. 1998. Enraizado de estacas juveniles en cinco especies de coníferas ornamentales: efecto del ácido indolbutírico AIB y de la temperatura. Rev. Ciencia Forestal Méx. 23: 29-38.

Melgares de Aguilar J, S Bañon, J Martínez, J Fernández, L Balenzategui. 2002. Influencia de diferentes temperaturas de sustrato y concentraciones de ácido indolbutírico en el esquejado de Coriaria myrtifolia. España. Actas de Horticultura, X Congreso Nacional de Ciencias Hortícolas. pp. 522-524.

Mesén F, A Newton, R Leakey. 1997. Vegetative propagation of Cordia alliodora (Ruiz et Pavón) Oken: the effects of IBA concentration, propagation medium and cutting origin. Forest Ecol. Manag. 92: 45-54.

Muñoz C. 1971. Chile, plantas en extinción. Santiago, Chile. Editorial Universitaria. 247 p.

Santelices R. 1993. Propagación vegetativa de raulí, roble y coihue a partir de estacas. Ciencia e Investigación Forestal (Chile) 7:37-48

Santelices R. 1998. Efecto de la temperatura del sustrato sobre el arraigamiento de estacas de canelo (Drimys winteri J.R et $\mathrm{G}$. Forster). Ciencias Forestales 12 (13): 27-32.

Santelices R, C García. 2003. Efecto del ácido indolbutírico y la ubicación de la estaca en el rebrote de tocón sobre la rizogénesis de Nothofagus alessandrii Espinosa. Bosque 24(2): 53-61.

Santelices R. 2005. Efecto del árbol madre sobre la rizogénesis de Nothofagus alessandrii. Bosque 26(3): 133-136.

Santelices R, A Cabello. 2006. Efecto del ácido indolbutírico, del tipo de la cama de arraigamiento, del sustrato, y del árbol madre en la capacidad de arraigamiento de estacas de Nothofagus glauca (Phil.) Krasser. Revista Chilena de Historia Natural 79: 55-64. 